\title{
Influencia de los factores sociodemográficos, familiares y el estado de la salud bucal en la calidad de vida de adolescentes peruanos
}

Influence of socio-demographic, familial factors and the state of oral health in the quality of life of Peruvian adolescents

Sonia Apaza-Ramos ${ }^{1, a}$, Gilmer Torres-Ramos ${ }^{2, a, b, c, d}$, Daniel José Blanco-Victorio ${ }^{3,4, a, e}$, Virginia Antezana$\operatorname{Vargas}^{3, a}$, Jeanette Montoya-Funegra ${ }^{1, a, b}$

\section{RESUMEN}

Objetivo: Evaluar la influencia de los factores familiares, sociodemográficos y el estado de salud bucal en la Calidad de Vida Relacionada a la Salud Bucal (CVRSB) de adolescentes peruanos. Materiales y métodos: En este estudio descriptivo y transversal se tomó una muestra aleatoria constituida por 131 adolescentes peruanos de 11 a 14 años de edad. El cuestionario de CVRSB utilizado fue el Child Perceptions Questionnaire $\left(\mathrm{CPQ}_{11}\right.$. $\left.{ }_{14}\right)$. Se registró el estado de salud bucal de los adolescentes como la presencia de caries dental a través del índice de piezas cariadas, perdidas y obturadas en dentición permanente (CPOD), sangrado gingival, trauma dental, defecto del esmalte y necesidad de tratamiento ortodóntico. Adicionalmente, se registró los factores familiares y sociodemográficos mediante un cuestionario para padres. Resultados: El modelo de regresión de Poisson mostró los predictores que influyen en la CVRSB de los adolescentes $(\mathrm{p}<0.05)$. También se encontraron correlaciones entre los puntajes bajos del $\mathrm{CPQ}_{11-14} \mathrm{y}$ cada dominio con la presencia de caries dental, sangrado gingival, el sexo femenino, el bajo ingreso familiar, no poseer una vivienda propia, no vivir con ambos padres, vivir en hacinamiento y el consumo de tabaco y/o alcohol $(\mathrm{p}<0.05)$. Conclusión: La CVRSB de los adolescentes peruanos evaluados se encuentra influenciada por diversos determinantes de la salud, no solo por las condiciones clínicas sino también por factores sociodemográficos y familiares en el cual está inmerso.

\section{PALABRAS CLAVE: Calidad de vida, salud bucal, adolescente. (DeCS, BIREME)}

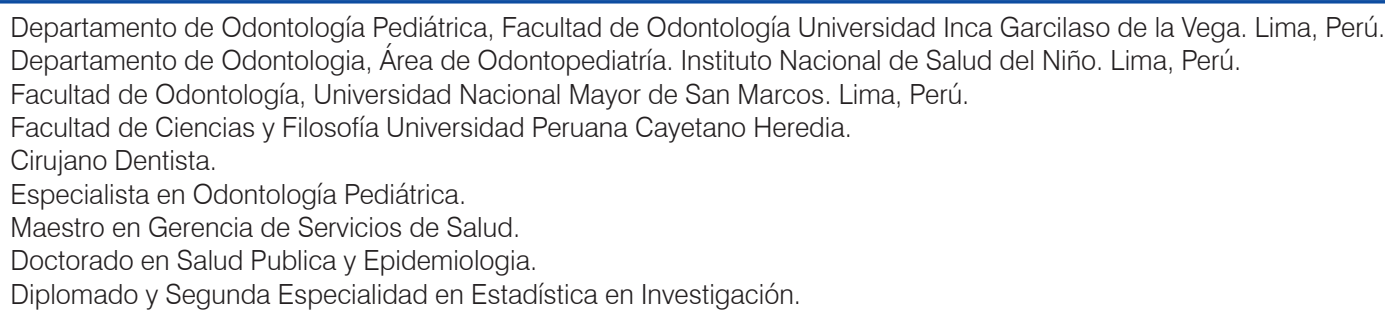




\section{SUMMARY}

Objectives: Evaluate the influence of socio-demographic, familial factors, and the state of oral health on the OHRQoL (Oral health-related quality of life) in Peruvian adolescents. Methods: For this descriptive transversal study, a random sample of 131 Peruvian adolescents aged 11 to 14 years was taken. What was used to gauge the CVRSB was The Child Perceptions Questionnaire $\left(C P Q_{11-14}\right)$. The state of oral health was registered as the dental caries through the decayed, missing and filled teeth (DMFT) index, bleeding gum, dental trauma, dental enamel hypoplasia, and orthodontic treatment need. Additionally, socio-demographic and familial factors were recorded through a questionnaire for parents. Results: The Poisson regression model showed predictors that influence the CVRSB on adolescents $(p<0.05)$. Correlations low scores on the $C P Q_{11-14}$ was also found, with some presence of dental caries, bleeding gum, considering also females, low family income, not having their own home, not living with both parents, living in overcrowding and consuming snuff and/or alcohol ( $p<0.05)$. Conclusion: The OHRQoL of Peruvian adolescents is influenced by several health determinants, not only for the clinical conditions but also by the socio-demographic and familial factors in which it is immersed.

\section{KEYWORDS: Quality of Life, oral health, adolescent. (MeSH, NLM)}

\section{INTRODUCCIÓN}

La Organización Mundial de la Salud (OMS) reconoce a la calidad de vida relacionada a la salud bucal (CVRSB) como una parte integral de la salud general y el bienestar. La CVRSB es un constructo multidimensional que incluye una evaluación subjetiva de la salud bucal de la persona, el bienestar funcional, el bienestar emocional, las expectativas y la satisfacción con la atención y el sentido de sí mismo (1).

En la práctica odontológica y la investigación, las medidas objetivas de la enfermedad proporcionan poca información acerca del impacto que generan los trastornos bucales sobre la vida diaria y la calidad de vida. Para llenar este vacío de información se han desarrollado múltiples estudios descriptivos en diferentes poblaciones, concluyendo que la mala salud bucal está relacionada con la mala calidad de vida (2). En la adolescencia esta relación se encuentra influenciada por diversos factores que comprometen la función, el bienestar y calidad de vida. Por ello, la salud bucal debe ser evaluada multidimensionalmente para orientar adecuadamente las políticas públicas de promoción y atención de la salud (3).

Desde las últimas décadas, se han venido realizado diversos estudios sobre la CVRSB en los adolescentes encontrándose asociación entre CVRSB y el estado de salud bucal $(4,5)$, el ambiente familiar, la educación de los padres (6), factores socioeconómico $(6,7)$ y hasta el aspecto psicosocial. Sin embargo, aún se necesita más investigación para evaluar el verdadero impacto de la CVRSB y la influencia de los tratamientos odontológicos en la percepción de la CVRSB (8).

Varios cuestionarios se han desarrollado para evaluar la percepción de la CVRSB en niños y adolescentes. Los más utilizados son the Child Perceptions Questionnaire (CPQ), the Child Oral Impacts on Daily Performances (C-OIDP) y the Child Oral Health Impact Profile (COHIP). Los tres cuestionarios parecen ser capaces de discriminar entre los grupos controles y los grupos con algún tipo de patología bucal (9).

EL $\mathrm{CPQ}_{11-14}$ es un cuestionario auto-percepción del impacto de las condiciones bucales y orofaciales en niños de 11 a 14 años de edad $(10,11)$. El $\mathrm{CPQ}_{11-14}$ ha sido ampliamente probado y fiable con varias versiones disponibles tales como la italiana (12), germana (13), danés (14), portugués de Brasil $(15,16)$, malayo de Brunéi, inglés de Nueva Zelanda (17) y camboyano (18). La versión en español del $\mathrm{CPQ}_{11-14}$ para la población peruana fue traducida $\mathrm{y}$ validada con satisfactorias propiedades psicométricas (19). En un estudio desarrollado en Lima, capital de Perú, se utilizó el $\mathrm{CPQ}_{11-14}$ donde se encontró que los adolescentes de 12 a 14 años, la experiencia de caries dental, presencias de TDI y la decoloración de la corona tuvieron un impacto negativo en el puntaje del $\mathrm{CPQ}_{11-14}$. Además, la maloclusión clase III mostró un impacto positivo en el dominio de bienestar emocional (20). 
A pesar de las recientes investigaciones sobre este tema en el Perú, aún quedan muchas dudas sobre la influencia del estado de salud bucal, factor económico, social y familiar en la CVRSB. Información importante para conocer las necesidades de tratamiento odontológico; y enfocar adecuadamente los programas de promoción de salud bucal y la atención clínica para la mejora de la calidad de vida de adolescente.

El objetivo de este estudio fue evaluar la influencia de los factores familiares, socioeconómicos y el estado de la salud bucal en la CVRSB de un grupo de adolescentes peruanos.

\section{MATERIALES Y MÉTODOS}

\section{Aspectos éticos}

El proyecto de investigación fue revisado $\mathrm{y}$ aprobado por la Universidad Inca Garcilaso de la Vega. Se garantizó la protección de los derechos de los participantes a través de un consentimiento informado dirigido hacia uno de los padres de familia o tutor apoderado del menor de edad, donde se detalló toda la información sobre la investigación, manifestándole su libre voluntad para participar o abandonar el estudio; y un asentimiento informado al adolescente. Además, se brindó confidencialidad guardando la identidad de todos los participantes del presente estudio.

\section{Población de estudio y tamaño de muestra}

El presente estudio descriptivo transversal fue realizado en los meses de mayo a junio del año 2014 en adolescentes de 11 a 14 años de la Institución Educativa Emblemática "José Granda", colegio público perteneciente a la jurisdicción del distrito de San Martin de Porres en la ciudad de Lima, capital del Perú. El distrito de San Martín de Porres destaca por ser el segundo distrito más poblado según reportes del Instituto Nacional de Estadística e Informática en el año 2013.

El cálculo del tamaño de muestra se efectuó a través de los resultados de una prueba piloto basados en la prevalencia de caries dental $(84.62 \%)$, con un intervalo de confianza del $95 \%$ y un error absoluto igual a 0.05 . Resultando un tamaño de muestra mínimo de 82 adolescentes, se seleccionaron aleatoriamente 148 adolescentes de 11 a 14 años de edad para compensar las posibles pérdidas por el rechazo de algunos adolescentes a participar. Los criterios de selección fueron: tener de 11 a 14 años de edad, no presentar ningún tipo de enfermedad sistémica, ni estar recibiendo algún tipo de tratamiento odontológico, sin tratamiento previo de ortodoncia y tener dentición permanente.

\section{Examen bucal de los adolescentes}

El examinador fue entrenado con imágenes de casos clínicos con las condiciones clínicas estudiadas en una semana por un especialista. Previamente, en un estudio piloto en 26 alumnos el examinador realizó exámenes bucales registrando todos los índices requeridos para el presente estudio, los participantes del estudio piloto fueron excluidos del estudio principal.

El registro de la caries dental se realizó a través del índice de cariados, perdidos y obturados en los dientes permanentes (CPOD); se evaluó la experiencia de caries dental dividiendo la muestra en dos grupos: con experiencia de caries dental $(\mathrm{CPOD}>0)$ y $\sin$ experiencia de caries dental $(\mathrm{CPOD}=0)$. Adicionalmente, se calculó la prevalencia de la caries dental y la severidad de la caries dental. De igual manera, se registró la ausencia o presencia del trauma dental, sangrado gingival y defectos del esmalte, todos los índices se evaluaron de acuerdo con los criterios diagnósticos de la OMS (21).

El índice de estética dental (IED) fue usado para evaluar la maloclusión y determinar la necesidad de tratamiento. Se analizaron los 10 componentes de IED, calculo el puntaje total del IED mediante su ecuación de regresión: ausencia $(\mathrm{x} 6)+$ apiñamiento + espaciamiento + diastema $(\mathrm{x} 3)+$ irregularidad superior + irregularidad inferior + resalte maxilar $(x 2)$ + resalte mandibular $(\mathrm{x} 4)+$ mordida abierta anterior $(\mathrm{x} 4)+$ relación molar anteroposterior $(\mathrm{x} 3)+13$; con el puntaje total del IED se registró la necesidad de tratamiento: no necesita tratamiento ortodóntico $(\mathrm{IED}<31)$ y necesita tratamiento ortodóntico $(\mathrm{IED} \geq 31)(22)$.

La evaluación clínica se realizó en la institución educativa en las aulas de clase, bajo luz natural; se 
utilizó instrumental estéril como: espejo dental, explorador, guantes estériles y sonda periodontal (OMS-621, Trinity).

\section{Medidores}

El $\mathrm{CPQ}_{11-14}$, evaluó la auto-percepción del niño sobre su calidad de vida relacionada a la salud bucal. Este cuestionario está compuesto por 36 preguntas, divididas en cuatro dominios: síntomas bucales (6 preguntas), limitaciones funcionales (9 preguntas), bienestar emocional ( 9 preguntas) y bienestar social (12 preguntas). Las puntuaciones de las respuestas estuvieron en escala de Likert: "nunca" $=0$, "una o dos veces" $=1$, "a veces" $=2$, "con frecuencia" $=3$ y "casi todos o todos los días" $=4$. Adicionalmente, tiene dos preguntas globales una sobre la auto-percepción de su salud bucal y la otra sobre la afectación de su salud bucal en su vida diaria. En el estudio piloto previo a la investigación, se determinó eliminar dos preguntas del $\mathrm{CPQ}_{11-14}$ (una del dominio de síntomas bucales $\mathrm{y}$ otra de limitaciones funcionales) para la mejora del alfa de Cronbach, y demostró ser comprendido satisfactoriamente por los adolescentes.

Se utilizó un cuestionario con opciones de respuestas dicotómicas para registrar los factores sociodemográficos y familiares que influyen en el adolescente, el cuestionario fue respondido por los padres de los adolescentes. El ingreso mensual de las familias para vivir en condiciones dignas es de tres remuneraciones mínimas (remuneración mínima= US \$240 por mes aproximadamente), mediante este parámetro se registró el ingreso familiar mensual $(\geq 3$ remuneraciones mínimos, o $<3$ salarios mínimos). De igual manera, se registró si la vivienda era propia, la educación de los padres (primaria completa o incompleta) y la ocupación de los padres (con empleo o sin empleo). Los factores familiares se evaluaron mediante cuatro preguntas: el niño vive con ambos padres, el número de hijos, el hacinamiento (1 persona, $\mathrm{o}>1$ persona por cuarto), y el consumo de tabaco y/o alcohol. Las preguntas se basaron en un estudio previo de Paula y col. (6).

\section{Análisis estadístico}

Todos los datos fueron procesados por el software Stata v13 (Stata Corporation, College Station, Texas). La consistencia interna del $\mathrm{CPQ}_{11-14}$ se determinó mediante el alfa de Cronbach. El $\mathrm{CPQ}_{11-14}$, los factores sociodemográficos, familiares y el estado de salud bucal se evaluaron mediante análisis descriptivos con pruebas no paramétricas de Mann-Whitney y Kruskal-Wallis, y las correlaciones a través del coeficiente de correlación de Spearman. El modelo de regresión de Poisson se asoció el puntaje total del $\mathrm{CPQ}_{11-14}$ y cada uno de sus dominios con los factores sociodemográficos, familiares y el estado de salud bucal; se considerado estadísticamente significativo a $\operatorname{los} \mathrm{p}<0.05$.

\section{RESULTADOS}

Participaron 148 adolescentes, de los cuales 17 fueron excluidos por no cumplir con alguno de los criterios de inclusión. Solo 131 adolescentes participaron, la edad promedio fue $12.6( \pm 1.2) \mathrm{y}$ la mediana (RIQ) fue 13 (2), la frecuencia y los porcentajes de todos los datos fueron registrados (Tabla 1).

El promedio del CPOD fue $2.29( \pm 2.26)$ y la mediana (RIQ) fue 2 (4), con un rango de puntaje observado entre 0 a 11 y la prevalencia de la caries dental según el CPOD fue del 70.99\% (n=93). Respecto a la autopercepción de la salud bucal de los adolescentes, el $42.75 \%$ considera que su salud bucal es buena (Figura 1) y referente a la percepción del efecto de la salud bucal en la vida cotidiana, el 64,1\% respondieron que si son afectados entre "poco" y "mucho" (Figura 2).

La consistencia interna obtenida por el $\mathrm{CPQ}_{11-14}$ fue buena, con un coeficiente de alfa de Cronbach de 0.82 para todo el puntaje del $\mathrm{CPQ}_{11-14}$ y vario por escalas siendo 0.81 para síntomas bucales, 0.76 para limitaciones funcionales, 0.73 para bienestar emocional y 0.77 para bienestar social. En las puntuaciones del $\mathrm{CPQ}_{11-14}$ y los dominios (Tabla 2), se observó que el dominio con menor promedio fue limitaciones funcionales y el mayor promedio fue de bienestar social.

Se observó que los promedios de todos los dominios y el $\mathrm{CPQ}_{11-14}$ (Tabla 3), se incrementan con la presencia de algún problema bucal como la caries dental y sangrado gingival. De igual manera, los factores sociodemográficos y familiares que incrementan el promedio son ser del sexo femenino, 
Tabla 1. Factores sociodemográficos, familiares y estado de la salud bucal de adolescentes $(n=131)$.

\begin{tabular}{|c|c|}
\hline Variables & N (\%) \\
\hline \multicolumn{2}{|l|}{ SALUD BUCAL } \\
\hline \multicolumn{2}{|l|}{ Caries dental - CPOD } \\
\hline Sin caries $C P O D=0$ & $38(29.01 \%)$ \\
\hline Con caries $C P O D>0$ & $93(70.99 \%)$ \\
\hline \multicolumn{2}{|l|}{ Trauma Dental } \\
\hline Ausencia & $124(94.66 \%)$ \\
\hline Presencia & $7(5.34 \%)$ \\
\hline \multicolumn{2}{|l|}{ Defectos del Esmalte } \\
\hline Ausencia & $96(73.28 \%)$ \\
\hline Presencia & $35(26.72 \%)$ \\
\hline \multicolumn{2}{|l|}{ Sangrado Gingival } \\
\hline Ausencia & $59(45.04 \%)$ \\
\hline Presencia & $72(54.96 \%)$ \\
\hline \multicolumn{2}{|c|}{ Necesidad de tratamiento ortodóntico } \\
\hline No necesita & $88(67.18 \%)$ \\
\hline Si necesita & $43(32.82 \%)$ \\
\hline \multicolumn{2}{|l|}{ SOCIODEMOGRÁFICO } \\
\hline \multicolumn{2}{|l|}{ Edad } \\
\hline 11 & $31(23.66 \%)$ \\
\hline 12 & $32(24.43 \%)$ \\
\hline 13 & $33(25.19 \%)$ \\
\hline 14 & $35(26.72 \%)$ \\
\hline \multicolumn{2}{|l|}{ Sexo } \\
\hline Masculino & $77(58.78 \%)$ \\
\hline Femenino & $54(41.22 \%)$ \\
\hline \multicolumn{2}{|l|}{ Ingreso Familiar } \\
\hline$\geq 3$ salarios mínimos & $30(22.90 \%)$ \\
\hline$<3$ salarios mínimos & $101(77.10 \%)$ \\
\hline \multicolumn{2}{|l|}{ Vivienda propia } \\
\hline$S i$ & $49(37.40 \%)$ \\
\hline No & $82(62.60 \%)$ \\
\hline \multicolumn{2}{|l|}{ Educación de la madre } \\
\hline Primaria completa & $116(88.55 \%)$ \\
\hline Primaria incompleta & $15(11.45 \%)$ \\
\hline \multicolumn{2}{|l|}{ Educación del padre } \\
\hline Primaria completa & $117(89.31 \%)$ \\
\hline Primaria incompleta & $14(10.69 \%)$ \\
\hline \multicolumn{2}{|l|}{ Ocupación de la madre } \\
\hline Con empleo & $71(54.20 \%)$ \\
\hline Sin empleo & $60(45.80 \%)$ \\
\hline \multicolumn{2}{|l|}{ Ocupación del padre } \\
\hline Con empleo & $121(92.37 \%)$ \\
\hline Sin empleo & $10(7.63 \%)$ \\
\hline \multicolumn{2}{|l|}{ FACTORES FAMILIARES } \\
\hline \multicolumn{2}{|l|}{ Niño vive con ambos padres } \\
\hline$S i$ & $79(60.31 \%)$ \\
\hline No & $52(39.69 \%)$ \\
\hline
\end{tabular}

$\begin{array}{cc}\text { Número de hijos } & \\ \text { 1 o } 2 \text { hijos } & 59(45.04 \%) \\ 3 \text { o mas } & 72(54.96 \%) \\ \text { Hacinamiento } & \\ \text { 1 persona por cuarto } & 50(38.17 \%) \\ >1 \text { persona por cuarto } & 81(61.83 \%) \\ \text { Consumo de tabaco y/o alcohol } & \\ \text { No } & 113(86.26 \%) \\ S i & 18(13.74 \%)\end{array}$

tener un ingreso $<3$ salarios mínimos, no vivir con ambos padres, vivir en hacinamiento y consumir tabaco y/o alcohol. Las variables que mostraron asociación con $\mathrm{CPQ}_{11-14}$ fueron la caries dental, el sexo, el hacinamiento y el consumir tabaco y/o alcohol. También se muestran las variables que mostraron asociación con cada dominio.

La relación entre los dominios del $\mathrm{CPQ}_{11-14} \mathrm{y}$ el CPOD (Figura 3), se puede evaluar gráficamente según linealidad (pendiente de la recta) se evidencia una pendiente mayor entre el domino bienestar social y CPOD interpretándose como una relación directa, siendo estadísticamente significativa, en comparación con los otros dominios (pendientes casi imperceptibles).

Las correlaciones de Spearman del $\mathrm{CPO}_{11-14} \mathrm{y}$ sus dominios con los factores sociodemográficos, familiares y el estado de la salud bucal (Tabla 4), mostraron una correlación baja pero significativa entre el bienestar emocional con el sexo y hacinamiento, interpretándose que cuando el puntaje de bienestar emocional aumenta la adolescente es mujer y vive en hacinamiento. De igual modo, cuando el puntaje del dominio de síntomas bucales aumenta el ingreso familiar es $<3$ salarios mínimos.

En el análisis del modelo de regresión de Poisson se ajustaron las variables individuales con la puntuación del $\mathrm{CPQ}_{11-14}$ y cada dominio (Tabla 5), donde solo se incluyeron las variables estadísticamente significativas. Se presenta un Modelo 1 (nulo) y un Modelo 2 ajustado. Después del ajuste de las covariables (Modelo 2), la presencia de sangrado gingival, el sexo femenino, el ingreso familiar $<3$ salarios mínimos y el consumo de tabaco y/o alcohol, se mostraron como determinantes del impacto 


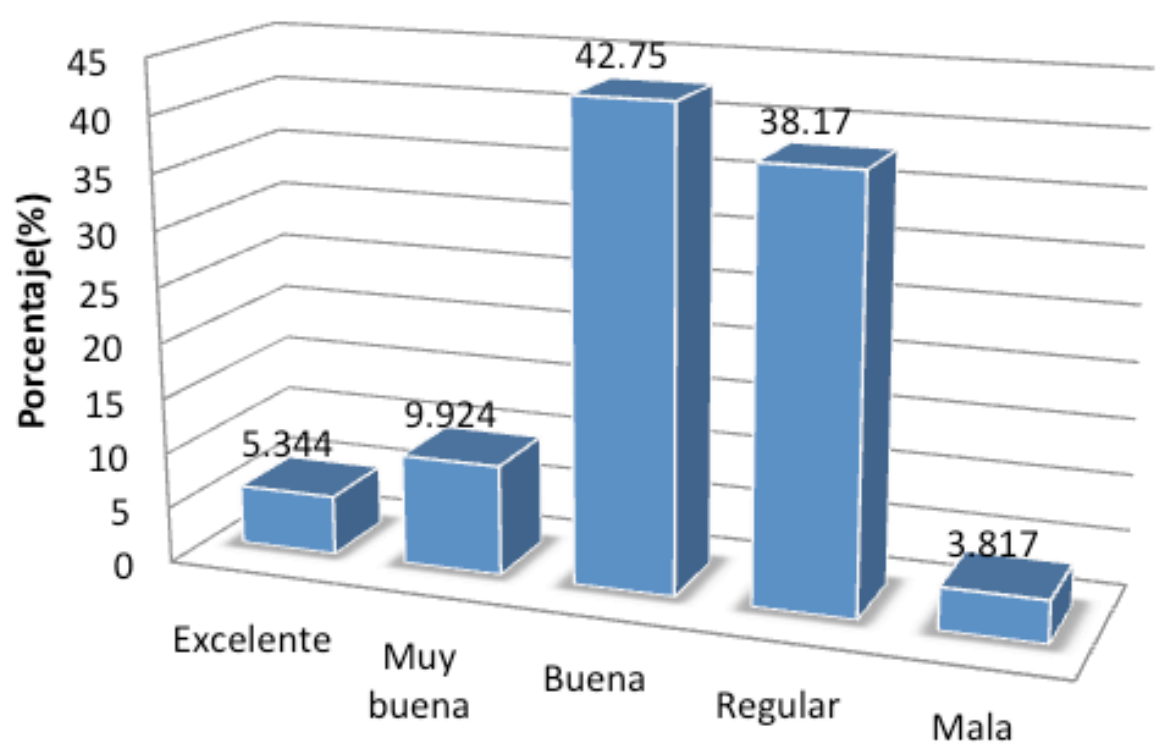

Figura 1. Autopercepción de la salud bucal de los adolescentes.

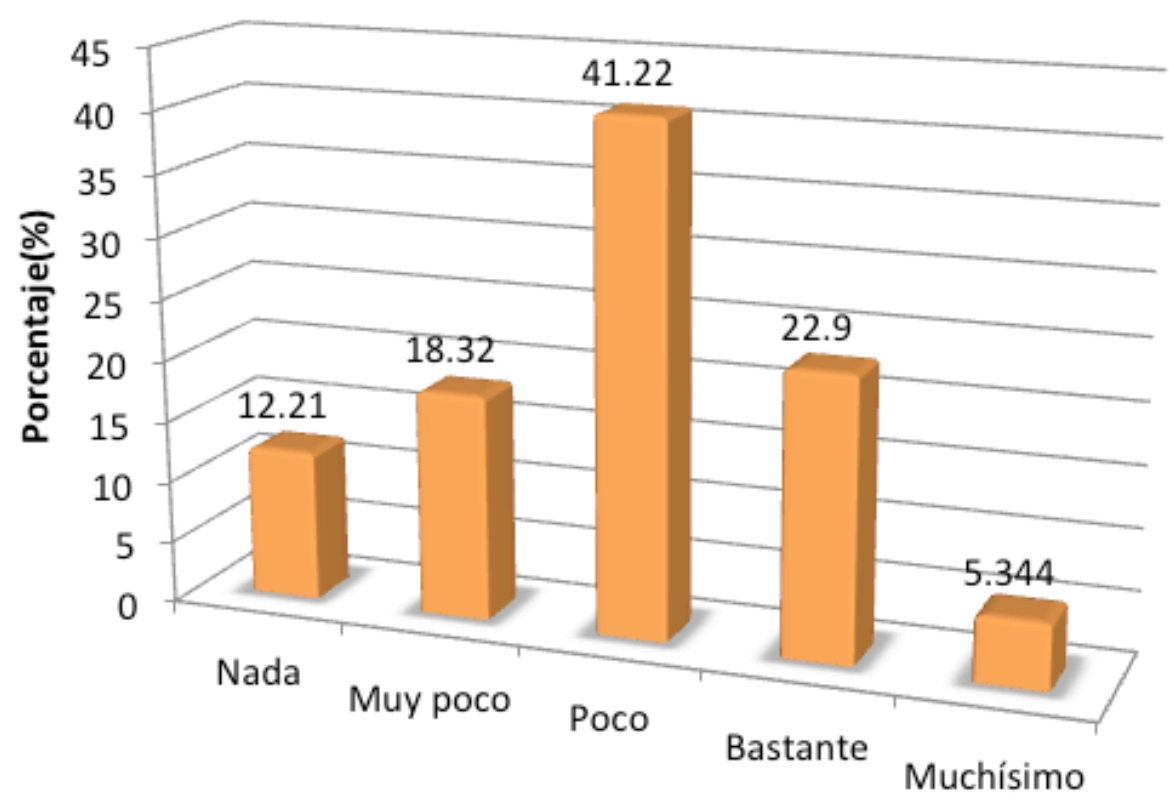

Figura 2. Percepción del efecto de la salud bucal en la vida cotidiana.

Tabla 2. Distribución descriptiva del $\mathrm{CPQ}{ }_{11-14}$ puntuación total y específica por dominio.

\begin{tabular}{cccccc}
\hline & Número de preguntas & Media & $\begin{array}{c}\text { Desviación } \\
\text { Estándar }\end{array}$ & $\begin{array}{c}\text { Rango } \\
\text { posible }\end{array}$ & Rango observado \\
\hline $\mathrm{CPQ}_{11-14}$ & 35 & 28.99 & 16.76 & $0-140$ & $3-83$ \\
Dominios & & & & & $0-16$ \\
Síntomas Bucales & 5 & 5.48 & 3.23 & $0-20$ & $0-25$ \\
Limitaciones Funcionales & 8 & 5.26 & 4.15 & $0-32$ & $0-32$ \\
Bienestar Emocional & 9 & 8.82 & 6.11 & $0-36$ & $0-30$ \\
Bienestar Social & 13 & 9.44 & 6.6 & $0-52$ & 0 \\
\hline
\end{tabular}


Tabla 3. Media del $\mathrm{CPQ}_{11-14}$ y dominios según los factores sociodemográficos, familiares y la salud bucal.

\begin{tabular}{|c|c|c|c|c|c|c|c|c|c|c|}
\hline \multirow[b]{2}{*}{ Variables } & \multicolumn{2}{|c|}{ Síntomas Bucales } & \multicolumn{2}{|c|}{$\begin{array}{l}\text { Limitaciones } \\
\text { Funcionales }\end{array}$} & \multicolumn{2}{|c|}{ Bienestar Emocional } & \multicolumn{2}{|c|}{ Bienestar Social } & \multicolumn{2}{|c|}{$\mathrm{CPQ}_{11-14}$} \\
\hline & $\begin{array}{l}\text { Media } \\
\text { (DS) }\end{array}$ & $\mathrm{p}^{*}$ & $\begin{array}{l}\text { Media } \\
\text { (DS) }\end{array}$ & $\mathrm{p}^{*}$ & $\begin{array}{l}\text { Media } \\
\text { (DS) }\end{array}$ & $\mathrm{p}^{*}$ & $\begin{array}{l}\text { Media } \\
\text { (DS) }\end{array}$ & $\mathrm{p}^{*}$ & Media (DS) & $\mathrm{p}^{*}$ \\
\hline \multicolumn{11}{|l|}{ SALUD BUCAL } \\
\hline \multicolumn{11}{|l|}{ Caries dental - CPOD } \\
\hline Sin caries $C P O D=0$ & $4.8(2.3)$ & \multirow{2}{*}{ NS } & $\begin{array}{c}4.3 \\
(3.1)\end{array}$ & \multirow{2}{*}{ NS } & $7.5(6.4)$ & \multirow{2}{*}{ NS } & $7.2(5.0)$ & \multirow[b]{2}{*}{0.020} & $23.8(13.4)$ & \multirow{2}{*}{0.031} \\
\hline Con caries $C P O D>0$ & $5.8(3.5)$ & & $\begin{array}{c}5.7 \\
(4.5)\end{array}$ & & $9.4(5.9)$ & & $10.3(7.0)$ & & $31.1(17.6)$ & \\
\hline \multicolumn{11}{|l|}{ Trauma Dental } \\
\hline Ausencia & $5.5(3.3)$ & \multirow{2}{*}{ NS } & $\begin{array}{c}5.3 \\
(4.2)\end{array}$ & \multirow{2}{*}{ NS } & $8.8(6.2)$ & \multirow{2}{*}{ NS } & $9.3(6.6)$ & & $29.0(16.8)$ & \multirow{2}{*}{ NS } \\
\hline Presencia & $4.9(3.0)$ & & $\begin{array}{l}4.0 \\
(3.2)\end{array}$ & & $8.7(5.6)$ & & $11.4(7.3)$ & NS & $29.0(17.5)$ & \\
\hline \multicolumn{11}{|l|}{ Defectos del Esmalte } \\
\hline Ausencia & $5.3(2.9)$ & \multirow{2}{*}{ NS } & $\begin{array}{c}4.9 \\
(4.0)\end{array}$ & \multirow{2}{*}{ NS } & $8.8(6.0)$ & \multirow{2}{*}{ NS } & $9.1(6.4)$ & & $28.1(16.2)$ & \multirow{2}{*}{ NS } \\
\hline Presencia & $6.1(4.0)$ & & $\begin{array}{c}6.4 \\
(4.5)\end{array}$ & & $8.8(6.4)$ & & $10.4(7.1)$ & 0.355 & $31.6(18.2)$ & \\
\hline \multicolumn{11}{|l|}{ Sangrado Gingival } \\
\hline Ausencia & $4.6(2.5)$ & \multirow{2}{*}{0.017} & $\begin{array}{c}4.7 \\
(3.0)\end{array}$ & \multirow{2}{*}{ NS } & $7.6(4.2)$ & \multirow{2}{*}{ NS } & $8.9(5.1)$ & & $25.9(10.8)$ & \multirow{2}{*}{ NS } \\
\hline Presencia & $6.2(3.6)$ & & $\begin{array}{c}5.7 \\
(4.9)\end{array}$ & & $9.8(7.2)$ & & $9.8(7.6)$ & NS & $31.5(20.1)$ & \\
\hline \multicolumn{11}{|c|}{$\begin{array}{l}\text { Necesidad de tratamiento } \\
\text { ortodóntico }\end{array}$} \\
\hline No necesita & $5.6(3.2)$ & & $\begin{array}{c}5.4 \\
(3.9)\end{array}$ & & $9.0(5.9)$ & & $8.6(5.8)$ & & $28.6(15.7)$ & \\
\hline Si necesita & $5.3(3.3)$ & NS & $\begin{array}{c}5.0 \\
(4.6)\end{array}$ & NS & $8.5(6.6)$ & NS & $11.0(7.8)$ & NS & $29.8(18.9)$ & NS \\
\hline SOCIODEMOGRÁI & & & & & & & & & & \\
\hline Edad & & & & & & & & & & \\
\hline 11 & $5.8(3.1)$ & & $\begin{array}{l}5.6 \\
(4.1)\end{array}$ & & $9.6(7.3)$ & & $9.1(6.3)$ & & $30.1(17.2)$ & \\
\hline 12 & $5.4(3.9)$ & & $\begin{array}{c}5.9 \\
(5.4)\end{array}$ & & $8.3(6.1)$ & & $10.0(6.8)$ & & $29.6(18.4)$ & \\
\hline 13 & $4.8(3.3)$ & NS** & $\begin{array}{c}4.1 \\
(3.6)\end{array}$ & NS** & $8.2(6.0)$ & NS** & $8.4(7.3)$ & NS** & $25.5(17.9)$ & NS** \\
\hline 14 & $5.9(2.6)$ & & $\begin{array}{c}5.5 \\
(3.2)\end{array}$ & & $9.2(5.1)$ & & $10.2(6.1)$ & & $30.8(13.6)$ & \\
\hline Sexo & & & & & & & & & & \\
\hline Masculino & $5.1(2.6)$ & & $\begin{array}{c}4.6 \\
(3.3)\end{array}$ & & $7.7(5.5)$ & & $8.8(6.6)$ & & $26.1(15.1)$ & \\
\hline Femenino & $6.0(3.9)$ & NS & $\begin{array}{c}6.3 \\
(5.0)\end{array}$ & NS & $10.4(6.6)$ & 0.012 & $10.4(6.6)$ & NS & $33.1(18.2)$ & 0.030 \\
\hline Ingreso Familiar & & & & & & & & & & \\
\hline$\geq 3$ salarios mínimos & $4.2(2.4)$ & & $\begin{array}{c}4.2 \\
(3.2)\end{array}$ & & $8.3(6.6)$ & & $7.2(4.8)$ & & $23.9(14.0)$ & \\
\hline$<3$ salarios mínimos & $5.9(3.3)$ & 0.014 & $\begin{array}{c}5.6 \\
(4.4)\end{array}$ & NS & $9.0(6.0)$ & NS & $10.1(6.9)$ & 0.047 & $30.5(17.3)$ & NS \\
\hline
\end{tabular}


$\mathrm{Si}$

No

Educación de la madre

Primaria completa

Primaria incompleta

Educación del padre

Primaria completa

Primaria incompleta

Ocupación de la madre

Con empleo

Sin empleo

Ocupación del padre

Con empleo

Sin empleo

$4.1(2.2)$

\section{FACTORES FAMILIARES}

Niño vive con ambos padres

Si

No

Número de hijos

1 o 2 hijos

3 o mas

Hacinamiento

1 persona por cuarto

$>1$ persona por cuarto

Consumo de tabaco y/o alcohol

No

$5.2(3.2)$

$7.1(3.1)$

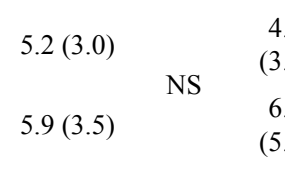

$5.1(3.0)$

$5.8(3.4)$

NS

5.2

(3.7)

5.3

(4.5)

$4.9(2.9)$

4.8

6.0
$(5.2)$
5.3

4.1)

5.2

(4.2)

NS

$7.9(7.0)$

$9.4(5.5)$

$9.1(6.3)$

NS

5.5

(2.9)

$6.8(3.9)$

NS

$9.0(6.1)$

NS

$29.4(17.0)$

(n)

$25.9(14.4)$

$8.6(5.8)$

$10.5(8.1)$

NS

$9.3(6.4)$

$10.8(8.3)$

NS

$28.7(16.1)$

$31.1(21.8)$

$8.8(6.6)$

NS

$9.2(7.1)$

$8.8(5.5)$

(4.5)

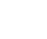

$9.7(6.0)$

NS

$28.8(17.6)$

$29.2(15.9)$

$8.8(6.3)$

NS

(2.1)

$9.1(3.7)$

NS

$9.5(6.7)$

8.7 (4.9)

NS

$29.2(17.2)$

NS

$26.7(10.5)$
NS

NS

NS

(3.3)

NS

$7.6(4.7)$

0.030

$8.3(5.7)$

$25.9(13.2)$

$10.6(7.5)$

$11.1(7.5)$

0.026

$33.7(20.3)$

NS

NS

$9.5(7.6)$

NS

$29.4(18.6)$

$9.4(5.7) \quad$ NS $\quad 28.7(15.2)$

NS

$8.2(4.9)$

$7.0(5.3)$

$8.4(7.0)$

NS

$24.9(15.9)$

0.010

$10.1(6.3)$

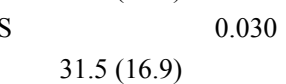

$9.9(6.3)$

(4.6)

$8.5(6.4)$

$9.2(6.7)$

NS

$28.0(16.9)$

0.034

$11.1(5.6)$

$35.2(14.4)$

*U Mann-Withney test

** H Kruskal-Wallis test

NS-No significativo

negativo de los dominios de síntomas bucales y limitaciones funcionales. Así mismo, la presencia de caries dental, la necesidad de tratamiento ortodóntico, el sexo femenino y no vivir con ambos padres son predictores del impacto negativo en los dominios de bienestar emocional y social. Los predictores de un impacto negativo para todo el $\mathrm{CPQ}_{11-14}$ son en el estado de salud bucal: la presencia de caries dental, defecto del esmalte y sangrado gingival; en los factores sociodemográficos: el sexo femenino, el ingreso familiar $<3$ salarios mínimos, primaria completa de la madre y padre con empleo; y en el factor familiar: 


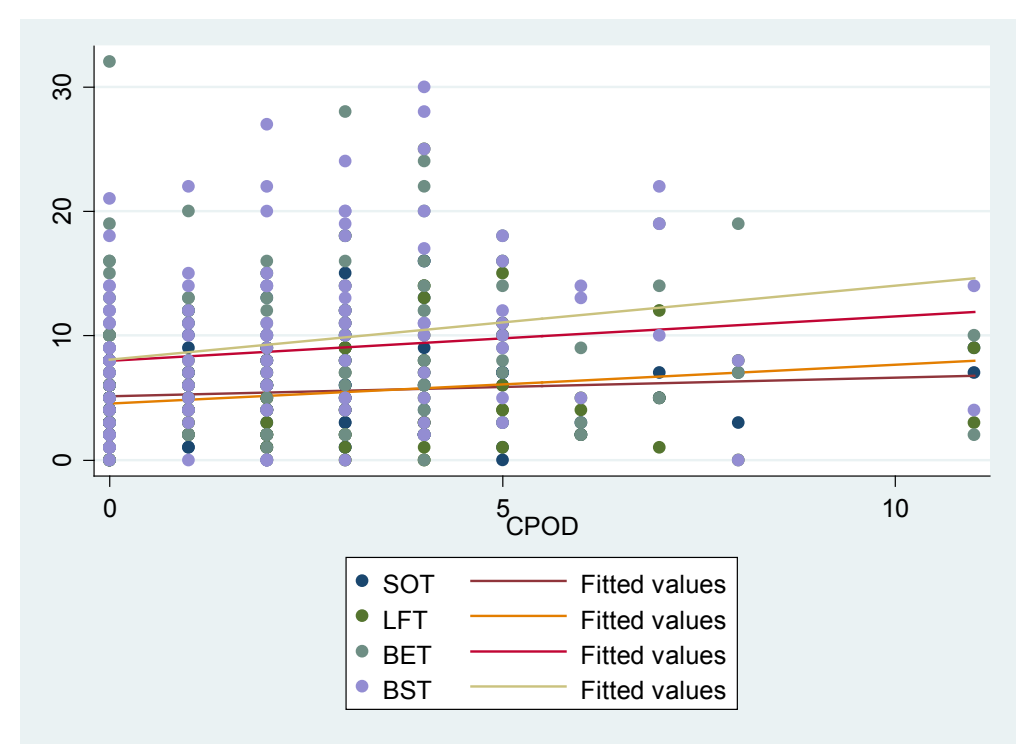

Figura 3. Diagrama de dispersión y linealidad según CPOD y dominios del $\mathrm{CPQ}_{11-14}$ en función al CPOD de los adolescentes.

Tabla 4. Correlación de Spearman del $\mathrm{CPO}_{11-14}$ y sus dominios con los factores sociodemográficos, familiares y el estado de la salud bucal.

\begin{tabular}{|c|c|c|c|c|c|}
\hline & $\begin{array}{c}\text { Síntomas Bucales } \\
\text { rho* }\end{array}$ & $\begin{array}{c}\text { Limitaciones } \\
\text { Funcionales } \\
\text { rho* }\end{array}$ & $\begin{array}{c}\text { Bienestar } \\
\text { Emocional } \\
\text { rho* }\end{array}$ & $\begin{array}{c}\text { Bienestar } \\
\text { Social } \\
\text { rho* }\end{array}$ & $\begin{array}{c}\text { CPQ } \\
11-14 \\
\text { rho* }\end{array}$ \\
\hline \multicolumn{6}{|l|}{ SALUD BUCAL } \\
\hline Caries dental - CPOD & 0.11 & 0.12 & 0.17 & $0.20 * *$ & $0.19^{* *}$ \\
\hline Trauma Dental & -0.04 & -0.06 & 0.01 & 0.08 & 0.01 \\
\hline Defectos del Esmalte & 0.07 & 0.15 & -0.01 & 0.08 & 0.08 \\
\hline Sangrado Gingival & $0.21^{* *}$ & 0.05 & 0.12 & 0.00 & 0.09 \\
\hline Necesidad de tratamiento ortodóntico & -0.05 & -0.08 & -0.06 & 0.14 & 0.01 \\
\hline \multicolumn{6}{|l|}{ SOCIODEMOGRÁFICO } \\
\hline Edad & 0.02 & -0.01 & 0.03 & 0.05 & 0.02 \\
\hline Sexo & -0.09 & -0.15 & $-0.22 * *$ & -0.12 & $-0.19^{* *}$ \\
\hline Ingreso Familiar & $0.22 * *$ & 0.12 & 0.06 & $0.17 * *$ & 0.16 \\
\hline Vivienda propia & 0.05 & -0.01 & $0.18^{* *}$ & 0.13 & 0.13 \\
\hline Educación de la madre & -0.15 & 0.06 & -0.10 & 0.01 & -0.05 \\
\hline Educación del padre & $-0.17 * *$ & 0.02 & 0.04 & 0.05 & 0.00 \\
\hline Ocupación de la madre & 0.00 & -0.05 & 0.04 & 0.07 & 0.03 \\
\hline Ocupación del padre & -0.13 & 0.01 & 0.05 & 0.01 & -0.01 \\
\hline \multicolumn{6}{|l|}{ FACTORES FAMILIARES } \\
\hline Niño vive con ambos padres & 0.10 & 0.07 & $0.19 * *$ & $0.19 * *$ & 0.17 \\
\hline Número de hijos & 0.07 & -0.03 & -0.05 & 0.06 & 0.01 \\
\hline Hacinamiento & 0.14 & 0.07 & $0.22 * *$ & 0.16 & $0.19^{* *}$ \\
\hline Consumo de tabaco y/o alcohol & $0.20^{* *}$ & 0.09 & $0.19 * *$ & 0.15 & $0.18^{* *}$ \\
\hline
\end{tabular}

*rho-Coeficiente de correlación de Spearman $* * \mathrm{p}<0.05$ 
Tabla 5. Asociación del $\mathrm{CPQ}_{11-14}$ y sus dominios con los factores sociodemográfico, familiares y el estado de la salud bucal a través del modelo de regresión de Poisson.

\begin{tabular}{|c|c|c|c|c|c|c|c|c|c|c|}
\hline & \multicolumn{2}{|c|}{ Síntomas Bucales } & \multicolumn{2}{|c|}{ Limitaciones Funcionales } & \multicolumn{2}{|c|}{ Bienestar Emocional } & \multicolumn{2}{|c|}{ Bienestar Social } & \multicolumn{2}{|c|}{$\mathrm{CPQ}_{11-14}$} \\
\hline & $\begin{array}{c}\text { Modelo } 1 \\
\text { (nulo) }\end{array}$ & $\begin{array}{l}\text { Modelo } 2 \\
\text { (completo) }\end{array}$ & $\begin{array}{c}\text { Modelo } 1 \\
\text { (nulo) }\end{array}$ & $\begin{array}{c}\text { Modelo } 2 \\
\text { (completo) }\end{array}$ & $\begin{array}{c}\text { Modelo } 1 \\
\text { (nulo) }\end{array}$ & $\begin{array}{l}\text { Modelo } 2 \\
\text { (completo) }\end{array}$ & $\begin{array}{c}\text { Modelo } 1 \\
\text { (nulo) }\end{array}$ & $\begin{array}{l}\text { Modelo } 2 \\
\text { (completo) }\end{array}$ & $\begin{array}{l}\text { Modelo } 1 \\
\text { (nulo) }\end{array}$ & $\begin{array}{c}\text { Modelo } 2 \\
\text { (completo) }\end{array}$ \\
\hline & $\begin{array}{c}\text { coef* }^{*} \\
{[95 \% \mathrm{CI}]}\end{array}$ & $\begin{array}{c}\text { coef* }^{*} \\
{[95 \% \mathrm{CI}]}\end{array}$ & $\begin{array}{c}\text { coef }^{*} \\
{[95 \% \mathrm{CI}]}\end{array}$ & $\begin{array}{c}\text { coef* }^{*} \\
{[95 \% \mathrm{CI}]}\end{array}$ & $\begin{array}{c}\text { coef* }^{*} \\
{[95 \% \mathrm{CI}]}\end{array}$ & $\begin{array}{c}\text { coef }^{*} \\
{[95 \% \mathrm{CI}]}\end{array}$ & $\begin{array}{c}\text { coef }^{*} \\
{[95 \% \mathrm{CI}]}\end{array}$ & $\begin{array}{c}\text { coef* }^{*} \\
{[95 \% \mathrm{CI}]}\end{array}$ & $\begin{array}{c}\text { coef }^{*} \\
{[95 \% \mathrm{CI}]}\end{array}$ & $\begin{array}{c}\text { coef }^{*} \\
{[95 \% \text { CI }]}\end{array}$ \\
\hline & $\begin{array}{c}1.70 \\
(1.63-1.77)\end{array}$ & & $\begin{array}{c}1.66 \\
(1.59-1.73)\end{array}$ & & $\begin{array}{c}2.18 \\
(2.12-2.23)\end{array}$ & & $\begin{array}{c}2.24 \\
(2.19-2.30)\end{array}$ & & $\begin{array}{c}3.37 \\
(3.34-3.40)\end{array}$ & \\
\hline \multicolumn{11}{|l|}{$\begin{array}{l}\text { SALUD } \\
\text { BUCAL }\end{array}$} \\
\hline Caries dental & & & & $\begin{array}{c}0.21 \\
(0.0 .3-0.39)\end{array}$ & & $\begin{array}{c}0.21 \\
(0.06-0.36)\end{array}$ & & $\begin{array}{c}0.22 \\
(0.08-0.37)\end{array}$ & & $\begin{array}{c}0.18 \\
(0.10-0.26)\end{array}$ \\
\hline Trauma Dental & & & & & & & & $\begin{array}{c}0.26 \\
(0.03-0.50)\end{array}$ & & \\
\hline $\begin{array}{l}\text { Defectos del } \\
\text { Esmalte }\end{array}$ & & & & $\begin{array}{c}0.27 \\
(0.11-0.43)\end{array}$ & & & & $\begin{array}{c}0.13 \\
(0.01-0.26)\end{array}$ & & $\begin{array}{c}0.07 \\
(0.00-0.15)\end{array}$ \\
\hline $\begin{array}{l}\text { Sangrado } \\
\text { Gingival }\end{array}$ & & $\begin{array}{c}0.25 \\
(0.10-0.40)\end{array}$ & & $\begin{array}{c}0.19 \\
(0.04-0.35)\end{array}$ & & $\begin{array}{c}0.24 \\
(0.12-0.37)\end{array}$ & & & & $\begin{array}{c}0.14 \\
(0.08-0.21)\end{array}$ \\
\hline $\begin{array}{l}\text { Necesidad de } \\
\text { tratamiento } \\
\text { ortodóntico }\end{array}$ & & & & & & $\begin{array}{c}-0.16 \\
(-0.29--0.03)\end{array}$ & & $\begin{array}{c}0.23 \\
(0.10-0.35)\end{array}$ & & \\
\hline \multicolumn{11}{|c|}{ SOCIODEMOGRÁFICO } \\
\hline \multicolumn{11}{|l|}{ Edad } \\
\hline Sexo & & $\begin{array}{c}-0.22 \\
(-0.43--0.07)\end{array}$ & & $\begin{array}{c}-0.36 \\
(-0.52--0.21)\end{array}$ & & $\begin{array}{c}-0.22 \\
(-0.34--0.10)\end{array}$ & & $\begin{array}{c}-0.19 \\
(-0.31--0.08)\end{array}$ & & $\begin{array}{c}-0.22 \\
(-0.29--0.16)\end{array}$ \\
\hline $\begin{array}{l}\text { Ingreso } \\
\text { Familiar }\end{array}$ & & $\begin{array}{c}0.32 \\
(0.13-0.51)\end{array}$ & & $\begin{array}{c}0.23 \\
(0.02-0.43)\end{array}$ & & & & $\begin{array}{c}0.23 \\
(0.07-0.39)\end{array}$ & & $\begin{array}{c}0.13 \\
(0.04-0.21)\end{array}$ \\
\hline $\begin{array}{l}\text { Vivienda } \\
\text { propia }\end{array}$ & & & & $\begin{array}{c}-0.19 \\
(-0.35--0.02)\end{array}$ & & & & & & \\
\hline $\begin{array}{l}\text { Educación de } \\
\text { la madre }\end{array}$ & & & & & & $\begin{array}{c}-0.32 \\
(-0.54--0.10)\end{array}$ & & & & $\begin{array}{c}-0.15 \\
(-0.26--0.03)\end{array}$ \\
\hline $\begin{array}{l}\text { Educación del } \\
\text { padre }\end{array}$ & & & & & & $\begin{array}{c}0.22 \\
(0.03-0.40)\end{array}$ & & & & \\
\hline \multicolumn{11}{|l|}{$\begin{array}{l}\text { Ocupación de } \\
\text { la madre }\end{array}$} \\
\hline $\begin{array}{l}\text { Ocupación del } \\
\text { padre }\end{array}$ & & & & & & & & $\begin{array}{c}-0.28 \\
(-0.51--0.05)\end{array}$ & & $\begin{array}{c}-0.23 \\
(-0.37--0.09)\end{array}$ \\
\hline \multicolumn{11}{|l|}{ FAMILIAR } \\
\hline $\begin{array}{l}\text { Niño vive con } \\
\text { ambos padres }\end{array}$ & & & & & & $\begin{array}{c}0.14 \\
(0.01-0.26)\end{array}$ & & $\begin{array}{c}0.24 \\
(0.11-0.36)\end{array}$ & & $\begin{array}{c}0.15 \\
(0.08-0.22)\end{array}$ \\
\hline $\begin{array}{l}\text { Número de } \\
\text { hijos }\end{array}$ & & & & & & $\begin{array}{c}-0.18 \\
(-0.30--0.05)\end{array}$ & & & & \\
\hline Hacinamiento & & & & & & $\begin{array}{c}0.30 \\
(0.16-0.44)\end{array}$ & & & & $\begin{array}{c}0.12 \\
(0.04-0.20)\end{array}$ \\
\hline $\begin{array}{l}\text { Consumo de } \\
\text { tabaco y/o } \\
\text { alcohol }\end{array}$ & & $\begin{array}{c}0.29 \\
(0.09-0.48)\end{array}$ & & $\begin{array}{c}0.29 \\
(0.07-0.50)\end{array}$ & & $\begin{array}{c}0.33 \\
(0.16-0.50)\end{array}$ & & & & $\begin{array}{c}0.25 \\
(0.16-0.35)\end{array}$ \\
\hline
\end{tabular}

*Coeficiente de la regresión de Poisson

no vivir con ambos padres, vivir en hacinamiento y consumir tabaco y/o alcohol.

\section{DISCUSIÓN}

El presente estudio evaluó la influencia de los factores sociodemográficos, familiares y el estado de la salud bucal en la CVRSB de adolescentes peruanos. Son escasos los estudios que relacionan los diferentes determinantes de la salud bucal y la calidad de vida en esta población, sabiendo que los países latinoamericanos como el Perú presentan una severidad de caries moderada (23) la cual se encuentra asociada con la pobreza (24). Sin embargo, en el presente estudio el nivel de severidad de caries dental fue bajo, con una prevalencia de $70.99 \%$. 
Por otro lado, gran parte de nuestra población percibió su salud bucal como "buena" o "regular" y cree que esta afecta "poco" o "bastante" su vida cotidiana (Figuras 1 y 2 ); esta información nos hace valorar la importancia de la salud bucal en la calidad de vida de los adolescentes. Por esta razón, es sumamente importante conocer los factores que influyen en la CVRSB del adolescente para poder orientar los programas y las estrategias de salud bucal en una población como la nuestra con alta necesidad de tratamiento odontológico y escasos programas de salud que cubran estos gastos.

La autopercepción de la CVRSB en el presente estudio según el $\mathrm{CPQ}_{11-14}$ obtuvo puntajes similares a otros estudios en adolescentes peruanos (20) y brasileros (25), pero mayores a los obtenidos en una población de adolescentes rusos (26) e italianos (12). Pudiéndose evidenciar que los latinoamericanos poseemos una mala CVRSB en comparación con otros países desarrollados como los europeos.

El modelo de regresión de Poisson ajustado mostró los factores que actúan como predictores influyendo de forma negativa en la CVRSB de los adolescentes. Se encontró asociación estadísticamente significativa de la caries dental con el $\mathrm{CPQ}_{11-14}$, los dominios de: limitaciones funcionales, bienestar emocional $\mathrm{y}$ social; resultados similares a los de Koposova y col. (26), en otros estudios en adolescentes brasileros se asoció con todo el $\mathrm{CPQ}_{11-14}$ con excepción del dominio de limitaciones funcionales (25).

En el presente estudio el trauma dental mostró asociación solo con el dominio de bienestar social similar al estudio de Pulache y col. (20); sin embargo, en un estudio en niños brasileros se encontró una asociación estadísticamente significativa del trauma dental con el $\mathrm{CPQ}_{11-14}$, el dominio de síntomas bucales, limitaciones funcionales y bienestar social (27). Por otro lado, en otros estudios no se encontró asociación con el $\mathrm{CPQ}_{11-14}(25)$.

Al igual que en un estudio en adolescentes brasileros (28), en el presente estudio se encontró una asociación estadísticamente significativa entre la presencia de defectos del esmalte y limitaciones funcionales; sin embargo, este estudio también encontro asociación con el bienestar emocional y el $\mathrm{CPQ}_{11-14}$
En el presente estudio la presencia de sangrado gingival estuvo asociada al $\mathrm{CPQ}_{11-14}$ y a todos los dominios excepto el de bienestar social; sin embargo, mostró asociación con el dominio de bienestar emocional y social en un estudio en adolescentes brasileros (6).

Estudio previos determinaron que los adolescentes peruanos presentan una maloclusión grave o muy grave según el IED; evidenciándose una alta prevalencia de maloclusiones, por lo que es necesario implementar programas para mejorar la salud bucal $(29,30)$. Según de Paula y col. (6) la necesidad de tratamiento ortodóntico está asociada al dominio de limitaciones funcionales; sin embargo, el presente estudio encontró que se encuentra asociada al bienestar emocional y social. Sin embargo, a pesar de las diferentes asociaciones estadísticamente significativas del estado de la salud bucal con el $\mathrm{CPQ}_{11-14}$; las correlaciones que se encontraron fueron la caries dental con el bienestar social y el $\mathrm{CPQ}_{11-14,} \mathrm{y}$ el sangrado gingival con los síntomas bucales.

El sexo femenino actuó como un predictor de una negativa CVRSB siendo estadísticamente significativo y mostrando correlación, resultados similares a estudios en adolescentes brasileros $(6,25)$. De igual modo, el ingreso familiar mensual estuvo asociado a una negativa CVRSB, con excepción al dominio de bienestar emocional; pero otros estudios encontraron una asociación con todos los dominios del $\mathrm{CPQ}_{11-14}$ (6). También se encontró asociaciones significativas en los adolescentes que no tenían una vivienda propia con el dominio de limitaciones funcionales y los padres con primaria incompleta con el dominio de bienestar emocional; en un estudio de Piovesan y col. (25) también se asoció la escolaridad del padre con la CVRSB.

Los factores familiares que mostraron asociaciones estadísticamente significativas y correlación para este estudio fueron no vivir con ambos padres con un impacto negativo en el $\mathrm{CPQ}_{11-14}$ y los dominios de bienestar, vivir en hacinamiento en el $\mathrm{CPQ}_{11-14}$ y el dominio de bienestar emocional, y el consumo de tabaco y/o alcohol en el $\mathrm{CPQ}_{11-14}$, el dominio de síntomas bucales, limitaciones funcionales y bienestar emocional. Los factores familiares muestran influencia en la CVRSB de los adolescentes debido a que estos forman parte de los determinantes de la 
salud (31).

El presente estudio se efectuó en una institución pública ubicada en un distrito populoso de Lima, con un nivel socioeconómico medio-bajo, reportando en general una pobre calidad de vida relacionada a la salud bucal. Para eliminar las disparidades encontradas en las condiciones de la salud bucal en comparación con otros países desarrollados, debemos integrar a la salud bucal en las políticas de los programas de salud. Así mismo, las escuelas odontológicas deben liderar comisiones de salud bucal dirigidas a la comunidad y a la enseñanza de los determinantes de la salud.

En conclusión, la CVRSB de los adolescentes peruanos de 11 a 14 años de edad se encuentra influenciada por diversos determinantes de la salud, no solo por las condiciones clínicas; sino también, por los factores sociodemográficos y familiares en el cual está inmerso.

\section{Correspondencia:}

\section{Sonia Apaza Ramos}

Av. Tomas Valle, Urb. San Pedro de Garagay Mz. "E" lote 18. Distrito San Martin de Porres. Lima, Perú. Correo electrónico: soniaapaza1@hotmail.com

\section{REFERENCIAS BIBLIOGRÁFICAS}

1. Sischo L, Broder HL. Oral health-related quality of life: what, why, how, and future implications. J Dent Res. 2011; 90(11):1264-70.

2. Allen PF. Assessment of oral health related quality of life. Health Qual Life Outcomes. 2003; 1:40.

3. Oliveira DC, Pereira PN, Ferreira FM, Paiva SM, Fraiz FC. Reported Impact of Oral Alterations on the Quality of Life of Adolescents: A Systematic Review. Pesq Bras Odontoped Clin Integr. 2013; 13(1):123-9.

4. Bakhtiar M, Mohammadi TM, Hajizamani A, Vossoughi M. Association of Oral Health Indicators with Qualityof-Life Related to Oral Health among Iranian Adolescent. J Int Oral Health. 2014; 6(6):5-9.

5. Naito M, Yuasa H, Nomura Y, Nakayama T, Hamajima N, Hanada N. Oral health status and healthrelated quality of life: a systematic review. J Oral Sci. 2006; 48(1):1-7.

6. De Paula JS, Leite IC, de Almeida AB, Ambrosano GM, Mialhe FL. The impacto of socioenvironmental characteristics on domains of oral health-related quality of life in Brazilian schoolchildren. BMC Oral Health. 2013; 13:10.
7. Peres $\mathrm{KG}$, Peres MA, Araujo CL, Menezes AM, Hallal PC. Social and dental status along the life course and oral health impacts inadolescents: a population-based birth cohort. Health Qual Life Outcomes. 2009; 7:95.

8. Tesch FC, Oliveira BH, Leão A. Measuring the impact of oral health problems on children's quality of life: conceptual and methodological issues. Cad Saude Publica. 2007; 23(11):2555-64.

9. Gilchrist F, Rodd H, Deery C, Marshman Z. Assessment of the quality of measures of child oral health-related quality of life. BMC Oral Health. 2014; 14:40.

10. Jokovic A, Locker D, Stephens M, Kenny D, Tompson B, Guyatt G. Validity and reliability of a questionnaire for measuring child oral-health-related quality of life. J Dent Res. 2002; 81(7):459-63.

11. Foster Page LA, Thomson WM, Jokovic A, Locker D. Validation of the Child Perceptions Questionnaire (CPQ 11-14). J Dent Res. 2005; 84(7):649-52.

12. Olivieri A, Ferro R, Benacchio L, Besostri A, Stellini E. Validity of Italian version of the Child Perceptions Questionnaire (CPQ11-14). BMC Oral Health. 2013; 13:55.

13. Bekes K, John MT, Zyriax R, Schaller HG, Hirsch C. The German version of the Child Perceptions Questionnaire (CPQ-G11-14): translation process, reliability, and validity in the general population. Clin Oral Investig. 2012; 16(1):165-71.

14. Wogelius P, Gjørup H, Haubek D, Lopez R, Poulsen S. Development of Danish version of child oral-healthrelated-related quality of lifequestionnaires (CPQ8-10 and CPQ11-14). BMC Oral Health. 2009; 9:11.

15. Goursand D, Paiva SM, Zarzar PM, Ramos-Jorge ML, Cornacchia GM, Pordeus IA, Allison PJ. Cross-cultural adaptation of the Child Perceptions Questionnaire 11-14 (CPQ $\left.{ }_{11-14}\right)$ for the Brazilian Portuguese language. Health Qual Life Outcomes. 2008; 6:2.

16. Barbosa TS, Tureli MC, Gavião MB. Validity and reliability of the Child Perceptions Questionnaires applied in Brazilian children. BMC Oral Health. 2009; 9:13.

17. Page LA, Thomson WM, Mohamed AR, Traebert J. Performance and cross-cultural comparison of the short-form version of the CPQ11-14 in New Zealand, Brunei and Brazil. Health Qual Life Outcomes. 2011; 9:40.

18. Turton BJ, Thomson WM, Foster Page LA, Saub R, Ishak AR. Responsiveness of the Child Perceptions Questionnaire 11-14 for Cambodian children undergoing basic dental care. Int J Paediatr Dent. 2015; 25(1):2-8.

19. Abanto J, Albites U, Bönecker M, Martins-Paiva 
S, Castillo JL, Aguilar-Gálvez D. Cross-cultural adaptation and psychometric properties of the Child Perceptions Questionnaire 11-14 (CPQ11-14) for the Peruvian Spanish language. Med Oral Patol Oral Cir Bucal. 2013; 18(6):e832-8.

20. Pulache J, Abanto J, Oliveira LB, Bönecker M, Porras JC. Exploring the association between oral health problems and oral healthrelated quality of life in Peruvian 11 to 14-yearold children. Int J Paediatr Dent. 2015;2. [Epub ahead of print]

21. WHO. Oral Health Surveys: Basics Methods, 5th ed. Geneva: Word Health Organization; 2013.

22. Cardoso CF, Drummond AF, Lages EM, Pretti H, Ferreira EF, Abreu MH. The Dental Aesthetic Index and dental health component of the Index of Orthodontic Treatment Need as tools in epidemiological studies. Int J Environ Res Public Health. 2011; 8(8): 3277-86.

23. Delgado-Angulo EK, Hobdell MH, Bernabé E. Poverty, social exclusion and dental caries of 12-year-old children: a cross-sectional study in Lima, Peru. BMC Oral Health. 2009; 9:16.

24. Petersen PE, Bourgeois D, Ogawa H, Estupinan-Day S, Ndiaye C. The global burden of oral diseases and risks to oral health. Bull World Health Organ. 2005; 83(9):661-9.

25. Piovesan C, Antunes JL, Guedes RS, Ardenghi TM. onomic and clinical factors on child oral healthrelated quality of life (COHRQoL). Qual Life Res. 2010; 19(9):1359-66.

26. Koposova N, Eriksen HM, Widstrãm E, Eisemann M, Opravin A, Koposov R. Oral health-related quality of life among 12-year-odls in Northern Norway and North-West Russia. Oral Health Dent Manag. 2012; 11(4):206-14.

27. Traebert J, Lacerda JT, Foster Page LA, Thomson WM, Bortoluzzi MC. Impact of traumatic dental injuries on the quality of life of schoolchildren. Dent Traumatol. 2012; 28(6):423-8.

28. Vargas-Ferreira F, Ardenghi TM. Developmental enamel defects and their impact on child oral health-related quality of life. Braz Oral Res. 2011; 25(6):531-7.

29. Bernabé E, Flores-Mir C. Orthodontic treatment need in Peruvian young adults evaluated through dental aesthetic index. Angle Orthod. 2006; 76(3):417-21.

30. Aliaga-Del Castillo A, Mattos-Vela MA, AliagaDel Castillo R, Del Castillo-Mendoza C. Malocclusions in children and adolescents from villages and native communities in the Ucayali Amazon region in Peru. Rev Peru Med Exp Salud Publica. 2011; 28(1):87-91.

31. Newton JT, Bower EJ. The social determinants of oral health: new approaches to conceptualizing and researching complex causal networks. Community Dent Oral Epidemiol. 2005; 33(1):25-34.

Recibido: $16 / 10 / 2014$

Aceptado: 18/03/2015 